Methods We investigated $102, \mathrm{DRB} 1 * 08$ positive, patients with JIA classified according to American Collage of Rheumatology criteria. As controls we selected 270, DRB1*08 positive, healthy individuals. We scanned $12 \mathrm{Mb}$ of DNA covering the HLA complex using the following microsatellite polymorphisms, from centromeric to telomeric: D6S291, DQCar, D6S273, MIB, D6S265, D6S2223 and D6S2239. An EM algorythm was used to estimate haplotypes and only the distributions of microsatellite alleles on DR8-DQ4 haplotypes was compared between patients and controls.

Results The microsatellite locus D6S265, which is located centromeric of HLA-A, showed a strong positive association with the disease for the ${ }^{*} 130 \mathrm{bp}$ allele $\left(\mathrm{OR}=30, \mathrm{p}<1^{-6}\right)$. In order to investigate whether this association could be explained by HLA-A2 the frequency of the HLA-A2 allele on DR8-DQ4D6S265*130 haplotype was compared among patients and controls. However, the D6S265*130 allele still showed evidence for disease association $\left(\mathrm{OR}=23, \mathrm{pp}<1^{-5}\right)$.

Conclusion The observed association of D6S265*130 allele with JIA is not secondary to the association of this allele with the DR8-DQ4-A2 haplotype. It shows that D6S265*130 allele could be a marker for additional predisposing factor involved in JIA, and HLA-A2 cannot explain all associations to the JIA in HLA class I region.

\section{OP0098 THE JAK3 PROMOTER REGION IS STIMULATED BY T CELL ACTIVATION AND CONTAINS ESSENTIAL AP-1 AND ETS-1 ELEMENTS}

${ }^{1} \mathrm{M}$ Aringer, ${ }^{2} \mathrm{DM}$ Frucht, ${ }^{2} \mathrm{M}$ Chen, ${ }^{2} \mathrm{M}$ Centola, ${ }^{2} \mathrm{JJ}$ O'Shea. ${ }^{1}$ Rheumatology/Internal Medicine III, University of Vienna, Austria, Wien, Austria; ${ }^{2}$ Arthritis and Rheumatism Branch, NIAMS/National Institutes of Health, Bethesda, MD, USA

10.1136/annrheumdis-2001.103

Background Janus Kinase 3 (Jak3) is a non-receptor tyrosine kinase essential for interleukin- (IL-) 2, 4, 7, 9, and 15 signalling. In $\mathrm{T}$ lymphocytes, Jak3 expressed is clearly increased upon $\mathrm{T}$ cell activation. The regulatory regions of the Jak3 promoter, however, are unknown.

Objectives We therefore decided to characterise the promoter region of Jak3.

Methods A 5'race procedure was used to detect the beginning of Jak3 cDNA derived from both the Jurkat $\mathrm{T}$ cell line and an NK cell line (NK3.3). All sequencing was performed on an ABI 377 automated sequencer using DNA amplified by PCR and the ABI Big Dye terminator kit. Putative promoter pieces were amplified by PCR and subcloned into a luciferase vector. In addition, site-directed mutagenesis was used to change two AP-1 sites and a putative Stat binding site within the core promoter region. Jurkat T cells were transfected both with these constructs and a beta-galactosidase vector for normalisation; both activities were sequentially read on a luminometer using the Tropix Dual Light kit.

Results All Jak3 mRNA started 5' of a 3515 basepair intron; the first basepair of the longest mRNA species found was denominated as +1 . The 1039 basepair DNA region 5 of the mRNA start $(-1013 /+27)$ worked as a (TATA-less) promoter and could be activated by PMA, PHA, or immobilised anti-CD3, but not anti-CD28. As expected, the promoter did not work in the reverse direction. Truncation constructs showed the active region situated within a 267 basepair $(-240 /+27)$ region, the core promoter was positioned between ?74 and ?16. Within this region, that contained also an SP-1 site, a double-AP1 site, but not the potential Stat site in between, was of major importance, as demonstrated by targeted point mutation. Surrounding the core promoter, two Ets-1 consensus elements had significant (around 6fold) enhancer activity.

Conclusion The Jak3 promoter is situated around 3500 basepairs 5 of its ATG-codon. The promoter can be activated by T cell receptor crosslinking and contains an active double AP-1 binding site and Ets- 1 consensus elements that work as significant enhancers. This may lead to improved IL-2 (or ?4, -7- or ? 15) signalling in disease states where $T$ lymphocytes are being activated.

\section{THU0001 TNF-ALPHA POLYMORPHISM AT POSITION -308 MIGHT PROTECT AGAINST RADIOGRAPHIC DAMAGE IN RA IRRESPECTIVE OF RHEUMATOID FACTOR OR HLA STATUS}

${ }^{1} \mathrm{C}$ Orellana, ${ }^{1} \mathrm{R}$ Sanmartí, ${ }^{1} \mathrm{JD}$ Cañete, ${ }^{2} \mathrm{~J}$ Yagüe, ${ }^{2} \mathrm{G}$ Ercilla, ${ }^{1} \mathrm{~A}$ Gómez, ${ }^{1} \mathrm{G}$ Salvador, 1J Muñoz-Gómez. 'Department of Rheumatology; ${ }^{2}$ Department of Immunology, Hospital Clinic, Barcelona, Spain

10.1136/annrheumdis-2001.104

\section{Background}

Objectives To assess the prevalence of TNF-alpha polymorphisms in patients with early rheumatoid arthritis (RA) in our population and investigate whether there is an association with radiographic severity.

Methods 68 patients with early RA (49F/19M, mean age $51 \pm$ $15 \mathrm{y}$, mean disease duration $15 \pm 13 \mathrm{mo}, \mathrm{RF}+72 \%$, mean number of tender joints at entry $6.3 \pm 5.7$, mean number of swollen joints at entry $8.23 \pm 7.33$ ). Patients were genotyped for HLADRB1 and TNF-alpha alleles. Hands and feet radiographs were taken at entry and then yearly during follow-up. The Larsen method was used to assess radiographic damage.

Results There were not differences in the distribution of TNFalpha alleles in the RA patients compared with controls. The TNF-alpha -308GG genotype instead of the ?308GA genotype was found to be more frequent in patients with no erosive disease at entry compared with those presenting erosions $(89 \%$ vs $62 \%, \mathrm{p}=0.0023)$. Patients not showing radiographic erosions during follow-up $(36.5 \pm 22 \mathrm{mo})$ also had more frequently the $308 \mathrm{GG}$ genotype (96\% vs. $74 \%$ in erosive disease, $\mathrm{p}=0.018$ ). There were no differences in disease duration nor follow-up between patients with erosive and non erosive disease. The TNF-alpha -308GG polymorphism was as frequent in patients with non erosive disease as in controls, and there were not differences either among RF+ve and RF-ve patients nor among patients with or without the shared epitope or who were HLADR4 positive. No other associations related to TNF-alpha were found in relation to radiographic damage.

Conclusion The presence of the ?308GG TNF-alpha genotype appears to be one of the factors related to less severe disease as determined by erosive disease, and may have a protective role compared with the ?308 GA genotype. 